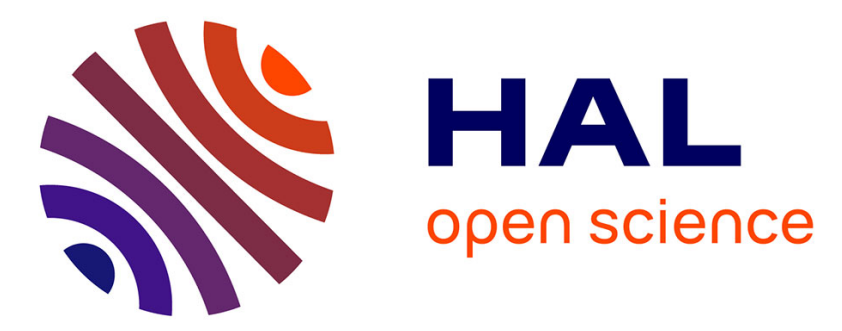

\title{
Lyapunov stabilization of discrete-time feedforward dynamics
}

\author{
Mattia Mattioni, Salvatore Monaco, Dorothée Normand-Cyrot
}

\section{To cite this version:}

Mattia Mattioni, Salvatore Monaco, Dorothée Normand-Cyrot. Lyapunov stabilization of discretetime feedforward dynamics. 57th IEEE Conference on Decision and Control (CDC 2018), Dec 2018, Miami Beach, FL, United States. pp.3471-3476. hal-01583221

\section{HAL Id: hal-01583221 \\ https://hal.science/hal-01583221}

Submitted on 19 Sep 2017

HAL is a multi-disciplinary open access archive for the deposit and dissemination of scientific research documents, whether they are published or not. The documents may come from teaching and research institutions in France or abroad, or from public or private research centers.
L'archive ouverte pluridisciplinaire HAL, est destinée au dépôt et à la diffusion de documents scientifiques de niveau recherche, publiés ou non, émanant des établissements d'enseignement et de recherche français ou étrangers, des laboratoires publics ou privés. 


\title{
Lyapunov stabilization of discrete-time feedforward dynamics
}

\author{
Mattia Mattioni ${ }^{1,2}$, Salvatore Monaco $^{1}$ and Dorothée Normand-Cyrot ${ }^{2}$
}

\begin{abstract}
The paper discusses stabilization of nonlinear discrete-time dynamics in feedforward form. First it is shown how to compute a Lyapunov function for the uncontrolled dynamics via the computation of a suitable cross-term. Then, stabilization is achieved in terms of $u$-average passivity. Several constructive cases are analyzed.

Index Terms-Lyapunov Methods; Stability of nonlinear systems; Algebraic/geometric methods
\end{abstract}

\section{INTRODUCTION}

Nonlinear discrete-time control theory has been attracting a growing interest in the control community for its impact into the sampled-data and, more in general, hybrid contexts. Although important works bridge the gap between the continuous-time and discrete-time domains through different methodologies (e. g., [1], [2], [3], [4], [5], [6], [7], [8],[9], [10]), hard difficulties represent an obstacle in extending results that are well-known and elegant in continuous time. Those issues are essentially concerned with the generic nonlinearity in the control variable of the dynamics and the difficulty to settle the geometric structure underlying its evolutions.

As a first attempt to characterize the accessibility properties of nonlinear discrete-time dynamics, the alternative differential-difference state-space representation (or $\left(F_{0}, G\right)$ form) was introduced in [11]. In this context, a discrete-time dynamics over $\mathbb{R}^{n}$ is described by two coupled differentialdifference equations

$$
\begin{aligned}
& x^{+}=F(x), \quad x^{+}:=x^{+}(0) \\
& \frac{\partial x^{+}(u)}{\partial u}=G\left(x^{+}(u), u\right)
\end{aligned}
$$

where (1a) models the free evolution described by a smooth mapping $F$ while (1a) the variational effect of the control via a complete vector field $G(\cdot, u) ; x^{+}(u)$ denotes a curve in $\mathbb{R}^{n}$ parametrized by $u \in \mathbb{R}$. Further exploiting this differential geometry framework, structural properties (e.g., invariance and decoupling, [12]) have been characterized up to introducing the concept of $u$-average passivity [13]. This latter notions enables to relax the necessity of a direct throughput as the usually required by passivity of discrete-time systems. At present, $u$-average passivity based controllers [14] (or

Mattia Mattioni thanks the Université Franco-Italienne/Università ItaloFrancese (UFI/UIF) for supporting his mobility from France to Italy within his $\mathrm{PhD}$ program.

${ }^{1}$ Dipartimento di Ingegneria Informatica, Automatica e Gestionale $A$. Ruberti (La Sapienza University of Rome); Via Ariosto 25, 00185 Rome, Italy \{mattia.mattioni, salvatore.monaco\}@uniroma1.it.

${ }^{2}$ Laboratoire des Signaux et Systèmes (L2S, CNRSCentraleSupelèc); 3, Rue Joliot Curie, 91192, Gif-sur-Yvette, France \{mattioni, cyrot\}al2s. centralesupelec.fr control Lyapunov design at large) can be discussed along these lines as exploited in the present paper with reference to stabilization of cascade dynamics.

More precisely, asymptotic stabilization of cascade discrete-time dynamics exhibiting an upper-triangular (or feedforward) form is addressed. Discrete-time forwarding design was firstly addressed in [15] via the construction of a bounded solution to a suitable control-dependent inequality so overcoming the difficulty of solving the nonlinear algebraic equation implicitly defining the feedback. In [16], it was extended to discrete-time systems by exploiting Immersion and Invariance in a quasi Lyapunov-based design where the a-priori knowledge of a Lyapunov function for the first-step is not required. In the present paper, we propose a two steps procedure based on control Lyapunov design and feedback passivation underlying the continuous-time counterpart ([17], [18]). Considering a two block cascade dynamics with nonlinear coupling mapping, a Lyapunov function is firstly constructed for the uncontrolled system via the computation of a suitable cross-term. Then, stabilization is achieved in terms of $u$-average passivity. Constructive solutions are discussed based on several specifications of the interconnection term. As a particular case, one recovers the case of dynamics in strict-feedforward form studied in [19] where the construction of a cross term reduces to the one of a coordinate transformation rendering the overall dynamics driftless. Finally, it is shown how these cascade connected forms intervene when representing input-delayed dynamics through dynamical extension. In this sense, we extend control Lyapunov design to discrete-time input delayed dynamics. A similar approach has been developed for dynamics issued from sampling [20] taking advantage of the primitive continuous-time properties. Work is progressing regarding multi-block cascade dynamics and analyzing the variety of control problems involving these structures.

The paper is organized as follows: in Section II, the existence of a cross-term is proven for uncontrolled dynamics while in Section III it is employed for stabilizing feedforward dynamics by means of $u$-average passivity. In Section IV some cases of study related to the connection term structure are discussed. In Section V conclusions are set.

\section{LYAPUNOV CROSS TERM FOR CASCADE DYNAMICS}

Consider a two block cascade dynamics of the form

$$
z_{k+1}=f\left(z_{k}\right)+\varphi\left(z_{k}, \xi_{k}\right), \quad \xi_{k+1}=a\left(\xi_{k}\right) .
$$

with $\xi \in \mathbb{R}^{n_{\xi}}, z \in \mathbb{R}^{n_{z}} ; f, \varphi$ and $a$ are continuous functions in their arguments and $(z, \xi)=(0,0)$ is an equilibrium state. Assuming that: 
A.1 $z_{k+1}=f\left(z_{k}\right)$ has a Globally Stable - GS - equilibrium at the origin with continuously differentiable, positive definite, radially unbounded Lyapunov function $W: \mathbb{R}^{n_{z}} \rightarrow \mathbb{R}_{\geq 0}$ such that $W(f(z))-W(z) \leq 0$;

A.2 $\xi_{k+1}=a\left(\xi_{k}\right)$ has a Globally Asymptotically Stable GAS - and Locally Exponentially Stable -LES- equilibrium at the origin with continuously differentiable, positive definite, radially unbounded Lyapunov function $U: \mathbb{R}^{n_{\xi}} \rightarrow \mathbb{R}_{\geq 0}$ such that $U(a(\xi))-U(\xi)<0$ for $\xi \neq 0$;

GS of the origin for the complete cascade cannot be a priori concluded. For this purpose, the further assumptions below are set so letting, in turn, to construct a Lyapunov function $V_{0}$ for the complete dynamics starting from the ones of each subsystem; namely, we assume the following:

A.3 the function $\varphi(z, \xi)$ satisfies the linear growth assumption; i.e. there exist $\mathscr{K}$-functions ${ }^{1} \gamma_{1}(\cdot), \gamma_{2}(\cdot)$ such that

$$
\|\varphi(z, \xi)\| \leq \gamma_{1}(\|\xi\|)\|z\|+\gamma_{2}(\|\xi\|) ;
$$

A.4 the function $W(z)$ verifies :

- given any $s(\cdot): \mathbb{R}^{n_{z}} \rightarrow: \mathbb{R}^{n_{z}}$ and $d(\cdot, \cdot): \mathbb{R}^{n_{z}} \times \mathbb{R}^{n_{\xi}} \rightarrow \mathbb{R}^{n_{z}}$

$$
|W(s(z)+d(z, \xi))-W(s(z))| \leq\left|\frac{\partial W}{\partial z} d(z, \xi)\right| ;
$$

- there exist $c, M \in \mathbb{R}_{>0}$ such that for $\|z\|>M$

$$
\left\|\frac{\partial W}{\partial z}\right\|\|z\| \leq c W(z) \text {. }
$$

By setting

$$
V_{0}(z, \xi)=W(z)+U(\xi)+\Psi(z, \xi)
$$

we aim at defining an additional continuous cross term $\Psi(z, \xi): \mathbb{R}^{n_{z}} \times \mathbb{R}^{n_{\xi}} \rightarrow \mathbb{R}$ to dominate the part with not definite sign when computing the difference

$$
\Delta_{k} V_{0}(z, \xi)=V_{0}\left(z_{k+1}, \xi_{k+1}\right)-V_{0}\left(z_{k}, \xi_{k}\right) .
$$

It is a matter of computations to verify that

$$
\begin{aligned}
\Delta_{k} V_{0}(z, \xi) & =\Delta_{k} U(\xi)+W\left(f\left(z_{k}\right)\right)-W\left(z_{k}\right) \\
& +W\left(f\left(z_{k}\right)+\varphi\left(z_{k}, \xi_{k}\right)\right)-W\left(f\left(z_{k}\right)\right)+\Delta_{k} \Psi(z, \xi)
\end{aligned}
$$

with $\Delta_{k} U(\xi)<0$ and $W\left(f\left(z_{k}\right)\right)-W\left(z_{k}\right) \leq 0$. It turns out that, for ensuring $\Delta_{k} V_{0}(z, \xi) \leq 0$, the cross term $\Psi(z, \xi)$ can be chosen to satisfy

$$
\Delta_{k} \Psi(z, \xi)=-W\left(f\left(z_{k}\right)+\varphi\left(z_{k}, \xi_{k}\right)\right)+W\left(f\left(z_{k}\right)\right)
$$

where the right hand side represents the part in $\Delta_{k} V_{0}$ whose sign is not definite. As a consequence, $\Psi(z, \xi)$ is defined as

$$
\Psi(z, \xi)=\sum_{k=0}^{\infty} W\left(f\left(z_{k}\right)+\varphi\left(z_{k}, \xi_{k}\right)\right)-W\left(f\left(z_{k}\right)\right)
$$

along the trajectories $\left(z_{k}, \xi_{k}\right)=(\tilde{z}(k, z, \xi), \tilde{\xi}(k, \xi))$ of (2) starting at $\left(z_{0}, \xi_{0}\right)=(z, \xi)$. The stability of the whole system follows from the existence of such function $V_{0}$.

\section{Theorem 2.1: Under assumptions A.1 to A.4}

\footnotetext{
${ }^{1}$ A function $\rho$ is said of class $\mathscr{K}$ if its continuous, strictly increasing and $\rho(0)=0$. It is said of class $\mathscr{K}_{\infty}$ if it is $\mathscr{K}$ and it is unbounded.
}

(i) $\quad \Psi: \mathbb{R}^{n_{z}} \times \mathbb{R}^{n_{\xi}} \rightarrow \mathbb{R}$ exists and is continuous;

(ii) $\quad V_{0}: \mathbb{R}^{n_{z}} \times \mathbb{R}^{n_{\xi}} \rightarrow \mathbb{R}$ in (3) is positive-definite and radially unbounded.

As a consequence the origin is a GS equilibrium of (2).

Proof: The proof is reported in Appendix.

Some constructive cases are discussed below in relation with the connection term $\varphi(z, \xi)$.

\section{A. Some particular cases}

1) Strict-feedforward dynamics: Consider strictfeedforward dynamics described by

$$
z_{k+1}=F z_{k}+\varphi\left(\xi_{k}\right), \quad \xi_{k+1}=a\left(\xi_{k}\right)
$$

with $\varphi(0)=0$ and $F^{\top} F=I$. In this case, Assumption A.1 is satisfied with $W(z)=z^{\top} z$ and A.4 is obviated. Specifying (4) for (6), one gets that the cross-term must satisfy

$$
\Delta_{k} \Psi(z, \xi)=-2 z_{k}^{\top} F^{\top} \varphi\left(\xi_{k}\right)-\varphi^{\top}\left(\xi_{k}\right) \varphi\left(\xi_{k}\right) .
$$

As a consequence $\Delta_{k} \Psi(z, \xi)=-\Delta_{k} W(z)$ and, according to (5), one computes

$$
\begin{aligned}
\Psi(z, \xi) & =\sum_{k=0}^{\infty}\left[z_{k+1}^{\top}(z, \xi) z_{k+1}(z, \xi)-z_{k}^{\top}(z, \xi) z_{k}(z, \xi)\right] \\
& =\left(z_{k}^{\top}(z, \xi) z_{k}(z, \xi)\right)_{\infty}-z^{\top} z
\end{aligned}
$$

where $\left(z_{k}^{\top}(z, \xi) z_{k}(z, \xi)\right)_{\infty}=\lim _{k \rightarrow \infty} z_{k}^{\top}(z, \xi) z_{k}(z, \xi)$ and $z_{k}(z, \xi)$ denotes the $z$-trajectory at time $k$ starting at $(z, \xi)$. According to (3), a Lyapunov function for (6) is thus

$$
V_{0}(z, \xi)=U(\xi)+\left(z_{k}^{\top}(z, \xi) z_{k}(z, \xi)\right)_{\infty} .
$$

More in detail, in this case (6) exhibits two invariant sets: a stable set where the evolutions are described by $\xi_{k+1}=a\left(\xi_{k}\right)$; a center set where the evolutions are described by $z_{k+1}=F z_{k}$. It is a matter of computations to verify that the projection of the trajectories of (6) onto the center set are provided by the mapping

$$
\phi(\xi)=-\sum_{\tau=k_{0}}^{\infty} F^{k_{0}-1-\tau} \varphi\left(\xi_{\tau}\right)
$$

verifying

$$
\phi\left(\xi_{k+1}\right)=F \phi\left(\xi_{k}\right)+\varphi\left(\xi_{k}\right) .
$$

Thus, under the coordinates change $\zeta=z-\phi(\xi)$, (6) is transformed into the decoupled dynamics

$$
\zeta_{k+1}=F \zeta_{k}, \quad \xi_{k+1}=a\left(\xi_{k}\right) .
$$

Hence, a Lyapunov function for the cascade is given by $\tilde{V}_{0}(\zeta, \xi)=U(\xi)+\zeta^{\top} \zeta$. Exploiting the strict-feedforward form, one easily verifies that the two Lyapunov functions $\tilde{V}_{0}$ and $\tilde{V}$ coincide up to a coordinates change.

Proposition 2.1: Let the strict-feedforward dynamics satisfy Assumptions A.1. Then, the Lyapunov function $V_{0}(z, \xi)=\tilde{V}_{0}(z+\phi(\xi), \xi)$ with $\phi(\xi): \mathbb{R}^{n_{\xi}} \rightarrow \mathbb{R}^{n_{z}}$ as in (9). As a consequence, the cross-term takes the form

$$
\Psi(z, \xi)=(\zeta-\phi(\xi))^{\top}(\zeta-\phi(\xi))-z^{\top} z .
$$


Proof: First, rewrite $\zeta^{\top} \zeta$ for $k_{0}=0$ as

$$
\begin{aligned}
& \left(z+\sum_{\tau=0}^{\infty} F^{-1-\tau} \varphi\left(\xi_{\tau}\right)\right)^{\top}\left(F^{k}\right)^{\top} F^{k}\left(z+\sum_{\tau=0}^{\infty} F^{-1-\tau} \varphi\left(\xi_{\tau}\right)\right) \\
& =\left\|z_{k}(z, \xi)+\sum_{\tau=0}^{\infty} F^{k-\tau-1} \varphi\left(\xi_{\tau}\right)-\sum_{\tau=0}^{k-1} F^{k-\tau-1} \varphi\left(\xi_{\tau}\right)\right\|^{2} .
\end{aligned}
$$

Because $\left(F^{k}\right)^{\top} F^{k}=I$ and

$$
z_{k}(z, \xi)=F^{k-k_{0}} z+\sum_{\tau=k_{0}}^{k-1} F^{k-\tau-1} \varphi\left(\xi_{\tau}\right)
$$

then, $\mathrm{b}$ letting $k \rightarrow \infty$, one gets

$$
\zeta^{\top} \zeta=\left(z_{k}^{\top}(z, \xi)\right)\left(z_{k}(z, \xi)\right)_{\infty} .
$$

Setting $\Psi(z, \xi)=(z-\phi(\xi))^{\top}(z-\phi(\xi))-z^{\top} z$, the cross term verifies (7) because of the invariance equality (10).

Remark 2.1: The cross-term in (8) depends on $\left\|z_{k}(z, \xi)\right\|^{2}$ that admits a limit for $k \rightarrow \infty$. This is not so in general for the solution $z_{k}(z, \xi)$, except in the particular case of $n_{z}=1$. $V_{0}(z, \xi)$ can be thus computed even if a decoupling change of coordinates does not exist.

2) $W(f(z)) \equiv W(z)$ : Let (2) verify A.1 with Lyapunov function $W(z)$ such that $W(f(z)) \equiv W(z)$. Then, (4) specifies as

$$
\Delta_{k} \Psi(z, \xi)=-W\left(f\left(z_{k}\right)+\varphi\left(z_{k}, \xi_{k}\right)\right)+W\left(z_{k}\right)=-\Delta_{k} W(z)
$$

so that the cross-term takes the form

$$
\Psi(z, \xi)=\sum_{k=0}^{\infty}\left[W\left(z_{k+1}\right)-W\left(z_{k}\right)\right]=W_{\infty}(z, \xi)-W(z)
$$

with $W_{\infty}(z, \xi):=\lim _{k \rightarrow \infty} W\left(z_{k}(z, \xi)\right)$. Consequently, one gets

$$
V_{0}(z, \xi)=U(\xi)+W_{\infty}(z, \xi) .
$$

3) $f(z)=z$ : When $f(z)=z$, one computes

$$
z_{\infty}(z, \xi)=z+\lim _{N \rightarrow \infty} \sum_{k=0}^{N} \varphi\left(z_{k}, \xi_{k}\right)
$$

and thus $W_{\infty}(z, \xi)=W\left(z_{\infty}(z, \xi)\right)$. Accordingly, the mapping $(z, \xi) \mapsto\left(z_{\infty}, \xi\right)$ defines a local coordinates change since

$$
\frac{\partial z_{\infty}}{\partial z}=I+\lim _{N \rightarrow \infty} \sum_{k=0}^{N} \frac{\partial \varphi}{\partial z}\left(z_{k}, \xi_{k}\right)
$$

and the sum vanishes at $\xi=0$. When the interconnection term $\varphi(\xi, z)$ does not depend on $z$, the above coordinates change is globally defined as one recovers a strictfeedforward form.

4) Particular structures of $\varphi(\xi)$ : When the coupling function $\varphi(\xi)$ is a finite polynomial of degree $p$, the crossterm is quadratic of degree $2 p$; the following example illustrates the case.

Example: Given

$$
z_{k+1}=z_{k}+\frac{3}{4} \xi_{k}^{2}, \quad \xi_{k+1}=\frac{1}{2} \xi_{k} .
$$

which verifies Assumptions A.1 to A.4 with $U(\xi)=\xi^{2}$ and $W(z)=z^{2}$, the coupling term $\varphi(\cdot)$ is assumed to be a finite polynomial of degree 4 . Hence, we set the cross-term in the form of a polynomial of degree 4

$$
\Psi(z, \xi)=a_{1} z \xi^{2}+a_{2} \xi^{4} .
$$

Accordingly, one computes $a_{1}, a_{2} \in \mathbb{R}$ to solve (7) that specialises as

$$
\begin{aligned}
& \frac{a_{1}}{2}\left(z+\frac{3}{4} \xi^{2}\right) \xi^{2}+\frac{a_{2}}{16} \xi^{4}-a_{1} z \xi^{2}-a_{2} \xi^{4} \\
= & \frac{1}{16} \xi^{4}+\frac{1}{2} \xi^{2}\left(z+\frac{3}{4} \xi^{2}\right)-\xi^{4}-2 z \xi^{2} .
\end{aligned}
$$

\section{Stabilization of EXTENDED CASCADE DYNAMICS}

In this section, the Lyapunov function $V_{0}(z, \xi)$ constructed above is exploited to show $u$-average passivity of the extended controlled cascade and compute the corresponding stabilizing feedback. Without loss of generality, the problem is set in the $\left(F_{0}, G\right)$ formalism (1).

\section{A. Feedforward dynamics}

Consider the two block controlled feedforward dynamics

$$
\begin{aligned}
z^{+} & =f(z)+\varphi(z, \xi), \quad z^{+}:=z^{+}(0) \\
\frac{\partial z^{+}(u)}{\partial u} & =G_{z}\left(z^{+}(u), \xi^{+}(u), u\right) \\
\xi^{+} & =a(\xi), \quad \xi^{+}:=\xi^{+}(0) \\
\frac{\partial \xi^{+}(u)}{\partial u} & =B_{\xi}\left(\xi^{+}(u), u\right)
\end{aligned}
$$

with uncontrolled part defined in (2) and controlled vector fields $G_{z}(\cdot, \cdot, u)$ and $B_{\xi}(\cdot, u)$. In a more compact way, one writes over $\mathbb{R}^{n_{z}} \times \mathbb{R}^{n_{\xi}}$

$$
x^{+}=F(x), \quad, \frac{\partial x^{+}(u)}{\partial u}=G\left(x^{+}(u), u\right), \quad x^{+}:=x^{+}(0)
$$

with $x=\operatorname{col}(z, \xi), F(x)=\operatorname{col}(f(z)+\varphi(z, \xi), a(\xi))$ and $G\left(x^{+}(u), u\right)=\operatorname{col}\left(G_{z}\left(z^{+}(u), B_{\xi}\left(\xi^{+}(u)\right), u\right)\right.$.

For any triplet $\left(z_{k}, \xi_{k}, u_{k}\right)$, by integrating (13b)-(13d) over $\left[0, u_{k}[\right.$ with initial condition (13a)-(13c), one recovers a feedforward dynamics in the form of a map

$$
\begin{aligned}
& z_{k+1}=f\left(z_{k}\right)+\varphi\left(z_{k}, \xi_{k}\right)+g\left(z_{k}, \xi_{k}, u_{k}\right) \\
& \xi_{k+1}=a\left(\xi_{k}\right)+b\left(\xi_{k}, u_{k}\right)
\end{aligned}
$$

where $\left(z_{k+1}, \xi_{k+1}\right)=\left(z^{+}\left(u_{k}\right), \xi^{+}\left(u_{k}\right)\right)$ and

$\frac{\partial g(z, \xi, u)}{\partial u}:=G_{z}\left(z^{+}(u), \xi^{+}(u), u\right), \frac{\partial b(\xi, u)}{\partial u}:=B_{\xi}\left(\xi^{+}(u), u\right)$.

Property 3.1: Given any $C^{1}$ function $S: \mathbb{R}^{n_{z}} \times \mathbb{R}^{n_{\xi}} \rightarrow \mathbb{R}$, one can rewrite

$$
S\left(x_{k+1}\right)=S\left(F\left(x_{k}\right)\right)+\int_{0}^{u_{k}} \mathrm{~L}_{G(\cdot, v)} S\left(x^{+}(v)\right) \mathrm{d} v
$$

where $\mathrm{L}_{G(\cdot, v)} S(x)$, denotes the usual Lie derivative of the function $S$ along $G(\cdot, v)$; i.e., $\mathrm{L}_{G(\cdot, v)} S(x):=\frac{\partial S}{\partial x} G(x, v)$. Furthermore, one has

$$
\int_{0}^{u_{k}} \mathrm{~L}_{G(\cdot, v)} S\left(x^{+}(v)\right) \mathrm{d} v=u_{k} \int_{0}^{1} \mathrm{~L}_{G\left(\cdot, \theta u_{k}\right)} S\left(x^{+}\left(\theta u_{k}\right)\right) \mathrm{d} \theta .
$$




\section{B. u-average passivity and $P B C$ design}

GAS of the equilibrium can now be achieved through $u$ average passivity-based control as introduced in [14]. The following definitions are recalled.

Definition 3.1 (u-average passivity, [14]): The dynamics (13), with output $y=H(x, u)$ is $u$-average passive with positive definite storage function $S(\cdot)$ if the following inequality holds for any $u \in \mathbb{R}$

$$
S\left(x^{+}(u)\right)-S(x) \leq \int_{0}^{u} H\left(x^{+}(v), v\right) \mathrm{d} v .
$$

Definition 3.2 (ZSD): Given (13) with output $H(x, u)$, let $Z \subset \mathbb{R}^{n_{z}} \times \mathbb{R}^{n_{\xi}}$ be the largest positively invariant set contained in $\left\{x \in \mathbb{R}^{n_{z}} \times \mathbb{R}^{n_{\xi}} \mid H(x, 0)=0\right\}$. (13) is ZeroState-Detectable (ZSD) if $x=0$ is asymptotically stable conditionally to $Z$.

Theorem 3.1: Consider (13) under A.1 to A.4, then:

- (13) is $u$-average passive with respect to the output

$$
H(z, \xi, u)=\mathrm{L}_{G(\cdot, u)} V_{0}(z, \xi)
$$

and storage function $V_{0}(z, \xi)$;

- if, furthermore, (13) with output $H(z, \xi, 0)$ is ZSD, the feedback $u_{d}$ solution

$$
u_{d}=-\frac{1}{u_{d}} \int_{0}^{u_{d}} \mathrm{~L}_{G(\cdot, v)} V_{0}\left(z^{+}(v), \xi^{+}(v), v\right) \mathrm{d} v
$$

achieves GAS of the equilibrium $(z, \xi)=(0,0)$;

- if the linear approximation of (13) is stabilizable then (16) ensures LES of the closed-loop.

Proof: $\quad$ Computing $\Delta_{k} V_{0}(z, \xi)=V_{0}\left(z_{k+1}, \xi_{k+1}\right)-$ $V_{0}\left(z_{k}, \xi_{k}\right)$ along the dynamics (13) one gets (dropping the $k$-index in the right hand side)

$$
\begin{gathered}
\Delta_{k} V_{0}(z, \xi)=U(a(\xi))-U(\xi)+\int_{0}^{u} \mathrm{~L}_{B_{\xi}(\cdot, v)} U\left(\xi^{+}(v)\right) d v \\
+W(f(z)+\varphi(z, \xi))-W(z)+\int_{0}^{u} \mathrm{~L}_{G_{z}\left(\cdot, \xi^{+}(v), v\right)} W\left(z^{+}(v)\right) d v \\
+\Psi(F(z, \xi))-\Psi(z, \xi)+\int_{0}^{u} \mathrm{~L}_{G(\cdot, v)} \Psi\left(z^{+}(v), \xi^{+}(v)\right) d v .
\end{gathered}
$$

By construction of $\Psi(\cdot)$ for $u=0$, one concludes $u$ average passivity with respect to the dummy output $H(\cdot, u)=$ $\mathrm{L}_{G(\cdot, u)} V_{0}$ and storage function $V_{0}$; i.e.

$$
\Delta_{k} V_{0}(z, \xi) \leq \int_{0}^{u} \mathrm{~L}_{G(\cdot, v)} V_{0}\left(z^{+}(v), \xi^{+}(v)\right) d v
$$

Accordingly, the control $u$ solution to (16) achieves GAS of the closed-loop equilibrium whenever (13) is ZSD with respect to $H(\cdot, 0)$. LES follows from $u$-average passivity plus the stabilizability of the linear approximation of (13) at the origin.

Remark 3.1: The damping controller $u_{d}$ solution of the equality (16) can equivalently be rewritten as the solution of

$$
u_{d}=-\int_{0}^{1} \mathrm{~L}_{G\left(\cdot, \theta u_{d}\right)} V_{0}\left(x^{+}\left(\theta u_{d}\right)\right) \mathrm{d} \theta .
$$

To avoid the difficult problem of solving implicit equalities, approximate solutions can be computed. In [16], the authors provide an explicit and exactly computable expression of the feedback $u$ which preserves $u$-average passivity and stability. The consequent solution is bounded by a positive constant $\mu \in \mathbb{R}$ and is defined as

$$
u_{\text {dap }}(x)=-K(x) \mathrm{L}_{G(\cdot, 0)} V_{0}\left(x^{+}(0)\right)
$$

for a suitable gain $K(\cdot)>0$.

Example: Consider the discrete-time system described by

$$
\begin{aligned}
& z^{+}=z+\xi, \quad \xi^{+}=\xi \\
& \frac{\partial z^{+}(u)}{\partial u}=\frac{1}{2}-\left(\xi^{+}(u)\right)^{2}, \quad \frac{\partial \xi^{+}(u)}{\partial u}=1
\end{aligned}
$$

or, equivalently,

$$
z_{k+1}=z+\xi+u\left(\frac{1}{2}-\xi^{2}\right)-u^{2} \xi-\frac{1}{3} u^{3}, \quad \xi_{k+1}=\xi+u
$$

which verifies Assumption A.1 with $W(z)=\frac{1}{2} z^{2}$ and Assumption A.2 with preliminary feedback $u=-\frac{2}{3} \xi$ and $U(\xi)=\frac{1}{2} \xi^{2}$. The cross term $\Psi(z, \xi)=\frac{1}{2}\left(z+\xi+\frac{\xi^{3}}{3}\right)^{2}-\frac{1}{2} z^{2}$ verifies $\Delta_{k} V_{0}(z, \xi)=\Delta_{k} U(\xi)=-\frac{4}{9} \xi_{k}^{2}$. Finally, the $u$-average output and the consequent control are provided by

$$
\begin{aligned}
& H(z, \xi, u)=4 \xi+\frac{3}{2} z+\frac{13}{8} u+\frac{1}{2} \xi^{3} \\
& u=-\frac{4}{7} z-\frac{32}{21} \xi-\frac{4}{21} \xi^{3} .
\end{aligned}
$$

\section{SOME CASES OF STUDY}

\section{A. The case of strict-feedforward dynamics}

Consider the controlled strict-feedforward dynamics

$$
\begin{aligned}
& z^{+}=F z+\varphi(\xi), \quad \frac{\partial z^{+}(u)}{\partial u}=G\left(\xi^{+}(u), u\right) \\
& \xi^{+}=a(\xi), \quad \frac{\partial \xi^{+}(u)}{\partial u}=B\left(\xi^{+}(u), u\right)
\end{aligned}
$$

or equivalently

$$
z_{k+1}=F z_{k}+\varphi\left(\xi_{k}\right)+g\left(\xi_{k}, u_{k}\right), \quad \xi_{k+1}=a\left(\xi_{k}\right)+b\left(\xi_{k}, u_{k}\right)
$$

with uncontrolled part (6) and by definition

$g\left(\xi_{k}, u_{k}\right):=\int_{0}^{u_{k}} G\left(\xi^{+}(v), v\right) \mathrm{d} v, \quad b\left(\xi_{k}, u_{k}\right):=\int_{0}^{u_{k}} B\left(\xi^{+}(v), v\right) \mathrm{d} v$

with $g(\cdot, 0)=0$ and $b(\cdot, 0)$. As already detailed, when $u \equiv 0$, the coordinates change $\zeta=z-\phi(\xi)$ in (9) transforms the system into the decoupled dynamics (11). Thus, by applying such coordinate change to (19), one gets

$$
\begin{array}{ll}
\zeta^{+}=F \zeta_{k}, & \frac{\partial \zeta^{+}(u)}{\partial u}=G_{\zeta}\left(\xi^{+}(u), u\right) \\
\xi^{+}=a\left(\xi_{k}\right), & \frac{\partial \xi^{+}(u)}{\partial u}=B\left(\xi^{+}(v), v\right) \mathrm{d} v
\end{array}
$$

where

$$
G_{\zeta}\left(\xi^{+}(u), u\right)=G\left(\xi^{+}(u), u\right)-\mathrm{L}_{B(\cdot, u)} \phi\left(\xi^{+}(u)\right) .
$$

As a consequence, Theorem 3.1 holds with average output

$$
Y_{1}(\zeta, \xi, u)=\mathrm{L}_{G_{\zeta}(\cdot u)} \tilde{V}_{0}(\zeta, \xi) .
$$


Remark 4.1: When $F=I$ and $n_{z}=1$, the coordinates change $\zeta=z-\phi(\xi)$ makes the $\zeta$-dynamics driftless. Accordingly, one recovers the result in [19] proposed when assuming directly in (19), $\xi_{k+1}=u_{k}$ and $n_{z}=1$.

Remark 4.2: In [16], the strict-feedforward stabilization is set in the Immersion and Invariance (I\&I) framework, [21] when $n_{z}=1$. Assuming A.2, a stable set over which the closed loop $\xi$-dynamics evolves is exhibited. The design aims at driving the off-stable set state components $\zeta$ to zero while ensuring boundedness of the full state trajectories. Moreover, I\&I is less demanding since the knowledge of a Lyapunov function $U(\xi)$ for the $\xi$-system is not necessary. On the other hand, the presented cross term approach covers a wider range of cases.

\section{B. Stabilization of input-delayed dynamics}

The result presented in the previous section is now applied to design $u$-average passivity-based controllers for discretetima systems affected by input delay. Consider the nonlinear input-delayed discrete-time dynamics

$$
z_{k+1}=f\left(z_{k}\right)+\varphi\left(z_{k}, u_{k-1}\right) .
$$

Setting the usual extension $\xi_{k}=u_{k-1}$, (22) rewrites as

$$
z_{k+1}=f\left(z_{k}\right)+\varphi\left(z_{k}, \xi_{k}\right), \quad \xi_{k+1}=u_{k}
$$

that clearly takes the form of (13) with $g(z, \xi, u)=0$ and $a(\xi)=0$. Assuming GS the origin of the dynamics $z_{k+1}=$ $f\left(z_{k}\right)$ with $C^{1}$ and radially unbounded Lyapunov function $W(z)$ and setting $U(\xi)=\xi^{2}$, the Lyapunov function $V_{0}(z, \xi)$ for (23) takes the form $V_{0}=\xi^{2}+W(z)+\Psi(z, \xi)$ with cross term solution of

$$
\left.\Delta_{k} \Psi(z, \xi)\right|_{u \equiv 0}=-W(f(z)+\varphi(z, \xi))+W(f(z)) .
$$

Under the assumptions in Theorem 3.1, one computes the output mapping $H_{d e l}(z, \xi)=\frac{\partial V_{0}}{\partial \xi}(z, \xi)$ with respect to which (23) is $u$-average passive so satisfying the inequality

$V_{0}(f(z)+\varphi(z, \xi), u)-V_{0}(z, \xi) \leq \int_{0}^{u} \frac{\partial V_{0}}{\partial \xi}(f(z)+\varphi(z, \xi), v) \mathrm{d} v$.

Accordingly, the control $u_{d e l}$ solution of the equality

$$
u_{d e l}=-\frac{1}{u_{d e l}} \int_{0}^{u_{d e l}} \frac{\partial V_{0}}{\partial \xi}(f(z)+\varphi(z, \xi), v) \mathrm{d} v
$$

stabilizes in closed-loop provided the ZSD property holds.

This comment can be generalized to multiple input delays and to a $z$-dynamics explicitly depending on $u$ as well. This is of peculiar interest when the problem of stabilizing a continuous-time time-delay system is set in the sampled-data context and reformulated as a discrete-time stabilizing one over an extended state space [22].

\section{Conclusions}

In this paper, we have addressed the problem of stabilizing discrete-time dynamics in feedforward form via Lyapunovbased and passivity-based methodologies. The study is detailed for the case of two interconnected dynamics by constructing a Lyapunov function via the definition of suitable cross-term. Work is progressing for a deeper understanding of the more general control problems that can be reconduced to the one here presented for cascade structures.

\section{REFERENCES}

[1] S. Monaco and D. Normand-Cyrot, "Nonlinear systems in discrete time," in Algebraic and geometric methods in nonlinear control theory. Springer, 1986, pp. 411-430.

[2] C. Mohtadi, "Bode's integral theorem for discrete-time systems," in IEE Proceedings D (Control Theory and Applications), vol. 137, no. 2. IET, 1990, pp. 57-66.

[3] L. Wei and C. Byrnes, "Design of discrete-time nonlinear control systems via smooth feedback," IEEE Trans. on Automatic Control, vol. 39, no. 11, pp. 2340-2346, 1994.

[4] D. Nešić, A. R. Teel, and E. D. Sontag, "Formulas relating kl stability estimates of discrete-time and sampled-data nonlinear systems," Systems \& Control Letters, vol. 38, no. 1, pp. 49-60, 1999.

[5] D. Kazakos and J. Tsinias, "The input-to-state stability condition and global stabilization of discrete-time systems," Automatic Control, IEEE Transactions on, vol. 39, no. 10, pp. 2111-2113, 1994.

[6] Z.-P. Jiang and Y. Wang, "Input-to-state stability for discrete-time nonlinear systems," Automatica, vol. 37, no. 6, pp. 857-869, 2001.

[7] E. M. Navarro-LóPez and E. Fossas-Colet, "Feedback passivity of nonlinear discrete-time systems with direct input-output link," Automatica, vol. 40, no. 8, pp. 1423-1428, 2004.

[8] N. Kazantzis, "A new approach to the zero-dynamics assignment problem for nonlinear discrete-time systems using functional equations," Systems \& control letters, vol. 51, no. 3, pp. 311-324, 2004.

[9] B. Jakubczyk, "Feedback linearization of discrete-time systems," Systems \& Control Letters, vol. 9, no. 5, pp. 411 - 416, 1987.

[10] P. Pepe, G. Pola, and M. Di Benedetto, "On lyapunov-krasovskii characterizations of stability notions for discrete-time systems with unknown time-varying time-delays," in Decision and Control (CDC), 2016 IEEE 55th Conference on. IEEE, 2016, pp. 447-452.

[11] S. Monaco and D. Normand-Cyrot, "A unified representation for nonlinear discrete-time and sampled dynamics," Journal of Mathematical Systems Estimation and Control, vol. 7, pp. 477-503, 1997.

[12] C. Califano, S. Monaco, and D. Normand-Cyrot, "Non-linear noninteracting control with stability in discrete-time: a geometric framework," International Journal of Control, vol. 75, no. 1, pp. 11-22, 2002.

[13] S. Monaco, D. Normand-Cyrot, and F. Tiefensee, "From passivity under sampling to a new discrete-time passivity concept," in Decision and Control, 2008. CDC 2008. 47th IEEE Conference on. IEEE, 2008, pp. 3157-3162.

[14] S. Monaco and D. Normand-Cyrot, "Nonlinear average passivity and stabilizing controllers in discrete-time," Systems \& Control Letters, vol. 60, pp. 431-439, 2011.

[15] F. Mazenc and H. Nijmeijer, "Forwarding in discrete-time nonlinear systems," International Journal of Control, vol. 71, no. 5, pp. 823-835, 1998.

[16] S. Monaco, D. Normand-Cyrot, and M. Mattioni, "Stabilization of feedforward discrete-time dynamics through immersion and invariance," in 2016 American Control Conference (ACC), July 2016, pp. 264-269.

[17] R. Sepulchre, M. Janković, and P. Kokotović, Constructive nonlinear control. Springer New York, 1997.

[18] L. Praly, R. Ortega, and G. Kaliora, "Stabilization of nonlinear systems via forwarding mod $\{\mathrm{L}$ g V $\}$," IEEE Transactions on Automatic Control, vol. 46, no. 9, pp. 1461-1466, 2001.

[19] S. Monaco and D. Normand-Cyrot, "Stabilization of nonlinear discrete-time dynamics in strict-feedforward form," in European Control Conference 13, 2013, pp. 2186-2191.

[20] M. Mattioni, S. Monaco, and D. Normand-Cyrot, "Sampled-data stabilization of feedforward dynamics with lyapunov cross-term," in 2016 IEEE 55th Conference on Decision and Control (CDC), Dec 2016, pp. 1322-1327.

[21] A. Astolfi and R. Ortega, "Immersion and invariance: a new tool for stabilization and adaptive control of nonlinear systems," IEEE Transactions on Automatic Control, vol. 48, no. 4, pp. 590-606, 2003.

[22] S. Monaco, D. Normand-Cyrot, and M. Mattioni, "Sampled-data stabilization of nonlinear dynamics with input delays through immersion and invariance," IEEE Transactions on Automatic Control, vol. PP, no. 99, pp. 1-1, 2016. 


\section{APPENDIX}

We begin the proof by showing (i). To this purpose, since the equilibrium of $\xi_{k+1}=a\left(\xi_{k}\right)$ is LES, we can write that for a real constant $|\alpha|<1$ and function $\gamma(\cdot) \in \mathscr{K}$, then $\tilde{\xi}(s, \xi) \leq$ $\gamma(\|\xi\|)|\alpha|^{s}$ for any $s \geq 0$.

From Assumption A.4 and this latter property, we write

$W\left(f\left(z_{k}\right)+\varphi\left(z_{k}, \xi_{k}\right)\right)-W\left(z_{k}\right) \leq$

$W\left(f\left(z_{k}\right)+\varphi\left(z_{k}, \xi_{k}\right)\right)-W\left(f\left(z_{k}\right)\right) \leq\left|\frac{\partial W}{\partial z} \varphi\left(z_{k}, \xi_{k}\right)\right| \leq$

$\left.\left.\left\|\frac{\partial W}{\partial z}\right\|(\gamma(\|\xi\|))|\alpha|^{k}+\gamma(\|\xi\|)\right)|\alpha|^{k}\left\|z_{k}\right\|\right) \leq c \gamma(\|\xi\|)|\alpha|^{k} W\left(z_{k}\right)$

Accordingly, $W(z)$ is not decreasing along the trajectories of (2) and $\left\|z_{k}\right\|$ and $\left\|\frac{\partial W}{\partial z}\left(z_{k}\right)\right\|$ are bounded on $[0, \infty)$ (because $W(z)$ is radially unbounded). Consequently, one can write

$$
W\left(f\left(z_{k}\right)+\varphi\left(z_{k}, \xi_{k}\right)\right)-W\left(f\left(z_{k}\right)\right) \leq \gamma_{1}(\|(z, \xi)\|) \alpha^{k}
$$

so getting that $W\left(f\left(z_{k}\right)+\varphi\left(z_{k}, \xi_{k}\right)\right)-W\left(f\left(z_{k}\right)\right)$ is summable over $[0, \infty)$ and (5) exists and is bounded for all $(z, \xi)$.

Continuity of (5) comes from the fact that it is the composition and the sum of continuous-function on $[0, \infty)$.

As far as (ii) is concerned, positive definiteness of $V_{0}$ is obtained by exploiting the radial unboundedness of $W(z)$.

$$
\begin{aligned}
& W\left(z_{k}\right)=W(z)+\sum_{t=0}^{k-1}\left[W\left(f\left(z_{t}\right)+\varphi\left(z_{t}, \xi_{t}\right)\right)-W\left(z_{t}\right)\right]= \\
& W(z)+\sum_{t=0}^{k-1}\left[W\left(f\left(z_{t}\right)+\varphi\left(z_{t}, \xi_{t}\right)\right)-W\left(f\left(z_{t}\right)\right)\right]+ \\
& \sum_{t=0}^{k-1}\left[W\left(f\left(z_{t}\right)\right)-W\left(z_{t}\right)\right]
\end{aligned}
$$

where the term $W\left(f\left(z_{t}\right)\right)-W\left(z_{t}\right)$ is non-increasing for any $t \geq 0$. By substracting both sides of the last equality by $W\left(f\left(z_{t}\right)\right)-W\left(z_{t}\right)$ and taking the limit for $k \rightarrow \infty$ one gets

$$
\begin{aligned}
& W_{\infty}(z)-\sum_{t=0}^{\infty}\left[W\left(f\left(z_{t}\right)\right)-W\left(z_{t}\right)\right]= \\
& W(z)+\sum_{t=0}^{\infty}\left[W\left(f\left(z_{t}\right)+\varphi\left(z_{t}, \xi_{t}\right)\right)-W\left(f\left(z_{t}\right)\right)\right]
\end{aligned}
$$

where $W_{\infty}(z)=\lim _{k \rightarrow \infty} W\left(z_{k}\right)$ and $\Psi(z, \xi)=\sum_{t=0}^{\infty}\left[W\left(f\left(z_{t}\right)+\right.\right.$ $\left.\left.\varphi\left(z_{t}, \xi_{t}\right)\right)-W\left(f\left(z_{t}\right)\right)\right]$. Hence, one gets that $V_{0}(z, \xi)$ rewrites as

$$
V_{0}(z, \xi)=W_{\infty}(z)-\sum_{t=0}^{\infty}\left[W\left(f\left(z_{t}\right)\right)-W\left(z_{t}\right)\right]+U(\xi) \geq 0 .
$$

From the radially unboundedness of $W$ and $U$ one has that if $V_{0}(z, \xi)=0$ then $\xi=0$. By construction, $V_{0}(z, 0)=$ $W(z)$ so concluding that $V_{0}(z, \xi)=0$ implies $(z, \xi)=(0,0)$. According to the last inequality this proves that $V_{0}$ is positivedefinite.

To prove its radial unboundedness we first point out that from (26) it follows that $V_{0}(z, \xi) \rightarrow \infty$ as $\|\xi\| \rightarrow \infty$ for any $z$. Hence, one has to show that

$$
\lim _{\|z\| \rightarrow+\infty}\left[W_{\infty}(z)-\sum_{t=0}^{\infty}\left(W\left(f\left(z_{t}\right)\right)-W\left(z_{t}\right)\right)\right]=+\infty .
$$

This will be achieved by lowerbounding (27) by means of a radially unbounded function deduced from $W(z)$. For, fix $\xi$ so that $\gamma(\|\xi\|)$ in (24) becomes a constant $C$. Accordingly, for any $k \geq 0$ we write

$$
\begin{aligned}
& \left|W\left(f\left(z_{k}\right)+\varphi\left(z_{k}, \xi_{k}\right)\right)-W\left(f\left(z_{k}\right)\right)\right| \leq \\
& \left\|\frac{\partial W}{\partial z}\right\|\left(C|\alpha|^{k}+C|\alpha|^{k}\left\|z_{k}\right\|\right) .
\end{aligned}
$$

It follows that

$$
\begin{aligned}
& W\left(f\left(z_{k}\right)+\varphi\left(z_{k}, \xi_{k}\right)\right)-W\left(f\left(z_{k}\right)\right) \geq \\
& -\left|W\left(f\left(z_{k}\right)+\varphi\left(z_{k}, \xi_{k}\right)\right)-W\left(f\left(z_{k}\right)\right)\right| \geq \\
& \geq-2\left\|\frac{\partial W}{\partial z}\right\| C|\alpha|^{k}\left\|z_{k}\right\|-C\left(1-\left\|z_{k}\right\|\right)\left\|\frac{\partial W}{\partial z}\right\||\alpha|^{k} .
\end{aligned}
$$

When $1-\left\|z_{k}\right\|>0$ the term $-C\left(1-\left\|z_{k}\right\|\right) \frac{\partial W}{\partial z} \||\alpha|^{k}$ can be discarded without affecting the inequality. On the other hand, when $1-\left\|z_{k}\right\| \leq 0$, it is bounded by $K_{2}|\alpha|^{k}$ so that

$$
\begin{aligned}
& W\left(f\left(z_{k}\right)+\varphi\left(z_{k}, \xi_{k}\right)\right)-W\left(f\left(z_{k}\right)\right) \geq \\
& -2\left\|\frac{\partial W}{\partial z}\right\| C|\alpha|^{k}\left\|z_{k}\right\|-K_{2}|\alpha|^{k} .
\end{aligned}
$$

Using A.4 we obtain

$W\left(f\left(z_{k}\right)+\varphi\left(z_{k}, \xi_{k}\right)\right)-W\left(z_{k}\right) \geq$

$\begin{cases}-K|\alpha|^{k} W\left(z_{k}\right)-K_{2}|\alpha|^{k}+W\left(f\left(z_{k}\right)\right)-W\left(z_{k}\right), & \|z\|>r \\ -K_{1}|\alpha|^{k} W\left(z_{k}\right)-K_{2}|\alpha|^{k}+W\left(f\left(z_{k}\right)\right)-W\left(z_{k}\right), & \|z\| \leq r\end{cases}$

with $r \geq 1$ and real $K, K_{1}, K_{2}$.

$\|z\|>r$ and $k \in[0, t)$

$$
\begin{gathered}
W\left(z_{k}\right) \geq \phi(k, 0) W(z)+\sum_{t=0}^{k-1} \phi(k-1, t)\left[-K_{2}\left|\alpha_{1}\right|^{t}+\right. \\
\left.W\left(f\left(z_{t}\right)\right)-W\left(z_{t}\right)\right]
\end{gathered}
$$

$\|z\| \leq r$ and $k \in[0, t)$

$W\left(z_{k}\right) \geq W(z)+\sum_{t=0}^{k-1}\left[-K_{1}|\alpha|^{t}-K_{2}|\alpha|^{t}+W\left(f\left(z_{t}\right)\right)-W\left(z_{t}\right)\right]$

with $\phi(k, t)=\prod_{j=t}^{k}\left(1-K|\alpha|^{j}\right)$. Accordingly, by mixing both the bounds, one gets

$W\left(z_{k}\right) \geq \phi(k, 0) W(z)+\sum_{t=0}^{k-1}\left(-K_{1}|\alpha|^{t}-K_{2}|\alpha|^{t}+W\left(f\left(z_{t}\right)\right)-W\left(z_{t}\right)\right)$

so that for all $k \geq 0, \phi(k, 0)$ admits a lower bound $K_{3}$ and

$$
W\left(z_{k}\right) \geq K_{3} W(z)+\sum_{t=0}^{k-1}\left[W\left(f\left(z_{t}\right)\right)-W\left(z_{t}\right)\right]+r_{k}
$$

with $r_{k}:=\sum_{t=0}^{k-1}\left[-K_{1}|\alpha|^{t}-K_{2}|\alpha|^{t}\right]$ which converges to a bounded solution $r^{*}$ over $[0, \infty)$. So, taking the limit when $k \rightarrow \infty$ one obtains

$$
W_{\infty}(z, \xi)-\sum_{t=0}^{k-1}\left[W\left(f\left(z_{t}\right)\right)-W\left(z_{t}\right)\right] \geq K_{3} W(z)+r^{*} .
$$

It is clear that $r^{*}$ and $K_{3}$ may depend on $\xi$ but are independent of $z$ so that (27) holds.

Accordingly, by construction $\left.V_{0}\left(z_{k+1}, \xi_{k+1}\right)-V_{0}\left(z_{k}, \xi_{k}\right)\right)=$ $W\left(f\left(z_{k}\right)\right)-W\left(z_{k}\right)+U\left(a\left(\xi_{k}\right)\right)-U\left(\xi_{k}\right) \leq 0$ so concluding the proof. 